\title{
THE AASK DATABASE V3.0: A Database of Human Experience during Aircraft Evacuation Incidents.
}

\author{
Galea E.R., Finney K.M., Dixon A.J.P., Cooney D.P. and Siddiqui A. \\ Fire Safety Engineering Group \\ University of Greenwich, \\ 30 Park Row, Greenwich, London SE10 9SL,UK \\ http://fseg.gre.ac.uk
}

\section{ABSTRACT}

This paper describes recent developments with the Aircraft Accident Statistics and Knowledge (AASK) database. The AASK database is a repository of survivor accounts from aviation accidents developed by the Fire Safety Engineering Group of the University of Greenwich with support from the UK CAA. Its main purpose is to store observational and anecdotal data from the actual interviews of the occupants involved in aircraft accidents. Access to the latest version of the database (AASK V3.0) is available over the Internet. AASK consists of information derived from both passenger and cabin crew interviews, information concerning fatalities and basic accident details. Also provided with AASK is the Seat Plan Viewer that graphically displays the starting locations of all the passengers - both survivors and fatalities - as well as the exits used by the survivors. Data entered into the AASK database is extracted from the transcripts supplied by the National Transportation Safety Board in the US and the Air Accident Investigation Branch in the UK. The quality and quantity of the data was very variable ranging from short summary reports of the accidents to boxes of individual accounts from passengers, crew and investigators. Data imported into AASK V3.0 includes information from 55 accidents and individual accounts from 1295 passengers and 110 crew.

Key words: aircraft accident, evacuation, database, evacuation data

\section{INTRODUCTION}

The AASK database is a repository of survivor accounts from aviation accidents [1-3]. Its main purpose is to store observational and anecdotal data from the actual interviews of the occupants involved in aircraft accidents. It was initially envisaged as an aid to the development of the airEXODUS aircraft evacuation model [4-8] where insight was required into how people actually behaved during evacuation from survivable aircraft crashes. However, the database has wider application to other areas of aviation safety, providing factual data regarding the evacuation process.

In the course of this study, it was found that, contrary to original expectations, a vast amount of human observational and anecdotal data was available. However, due to its nature, this data is difficult to secure and analyse. To aid in its storage and analysis, the computer based relational database AASK was developed [1,2]. This paper describes the latest release of the database, AASK V3.0 [3]. In developing V3.0 of AASK two issues were tackled, first was the inclusion of additional data and the second concerned improving the capability and functionality of the database. The data held in AASK V2.0 $[1,2]$ related to details from 34 accidents and passenger information from 772 survivors. Two types of additional data was provided. Firstly, additional information was obtained 
relating to accidents and passengers already existing in the database. Secondly, some 21 new accidents were added to AASK providing a total of 55 accidents and accounts from 1,295 passengers. The data is taken from accidents that occurred between $4 / 4 / 77$ and $8 / 3 / 98$.

In terms of the functionality and capability of the database, AASK V3.0 has been made available to users over the internet. The query engine developed for AASK has been designed so that users without a detailed understanding of the ACCESS database - on which AASK is based - can easily make use of the data. It should be stressed however that to run meaningful queries the user must understand the nature of the data held in the database. The cabin crew component has become a significant aspect of the database providing insight into cabin conditions and passenger behaviour as seen from professionally trained cabin specialists. In addition, 329 records of passenger/crew fatalities have been added. The Seat Plan Viewer graphically displays the starting locations of all the passengers - both survivors and fatalities - as well as the exits used by the survivors.

\section{AASK DATABASE}

AASK V3.0 consists of five main components the, (a) User Interface, (b) Data Viewer, (c) Seat Plan Viewer, (d) Data Query interface, and (e) Data Entry interface. It should be noted that in order to preserve the integrity of the data at the heart of AASK, data entry is restricted to the data base manager (initially the database developers). General users of AASK will only have access to view the data and to launch queries. Details of the AASK database are described in [3], here only a brief description of the key components of the database are provided.

\subsection{The Data Viewer}

The Data Viewer allows users to view all of the data records available within AASK. For convenience this is split into four sections each dealing with a different aspect of the accident.

\subsubsection{Accident Component}

The Accident Component is key to the whole structure of AASK and is a reference point for all the other components and data. No passengers, cabin crew or fatalities can be entered until the accident details are entered in this part of the database. In common with the rest of AASK V3.0, it is possible to enter an accident into the accident database where only part of the required information is available i.e. it is not necessary to complete all the fields in the accident database. There are a maximum of 20 fields possible including an ability to enter a brief accident summary.

It should be stressed that AASK V3.0 is not an accident database and this component has a minimum of factual data relating to each incident. The emphasis here is on passenger and crew data and not the technical aspects of the accident. By using common formats AASK V3.0 is able to link to the SAD [8] database for further analysis concerning accident details. 


\subsubsection{Passenger Component}

There are currently 1295 passenger records in AASK V3.0, each with a possible 69 places to enter data including general fields and notes. The passenger component is organised into a series of forms to ease data entry. The design allows similar data to be grouped and entered together. Depicted in Figure 1 is the "Basic Passenger Info" form. Most forms also contain several places where additional notes can be stored. Details listed include the reaction to the call to evacuate, the presence of travelling companions, difficulties experience with seat belts, etc.

\subsubsection{Cabin Crew Component}

The Cabin Crew component consists of 110 cabin crew accounts. There are 275 fields stored for each crew member covering aspects such as pre-flight briefing, observations of queuing behaviour and search and sweep operations. The data base provides opportunities to cross check between crew accounts and in certain instances to check the validity of passenger accounts.

\subsubsection{Fatalities Component}

The smallest of all the components in the AASK is the Fatalities Database. This stores information concerning any fatalities (passengers, cabin or flight crew) that are reported. AASK V3.0 currently holds records for 327 fatalities each with 16 fields.

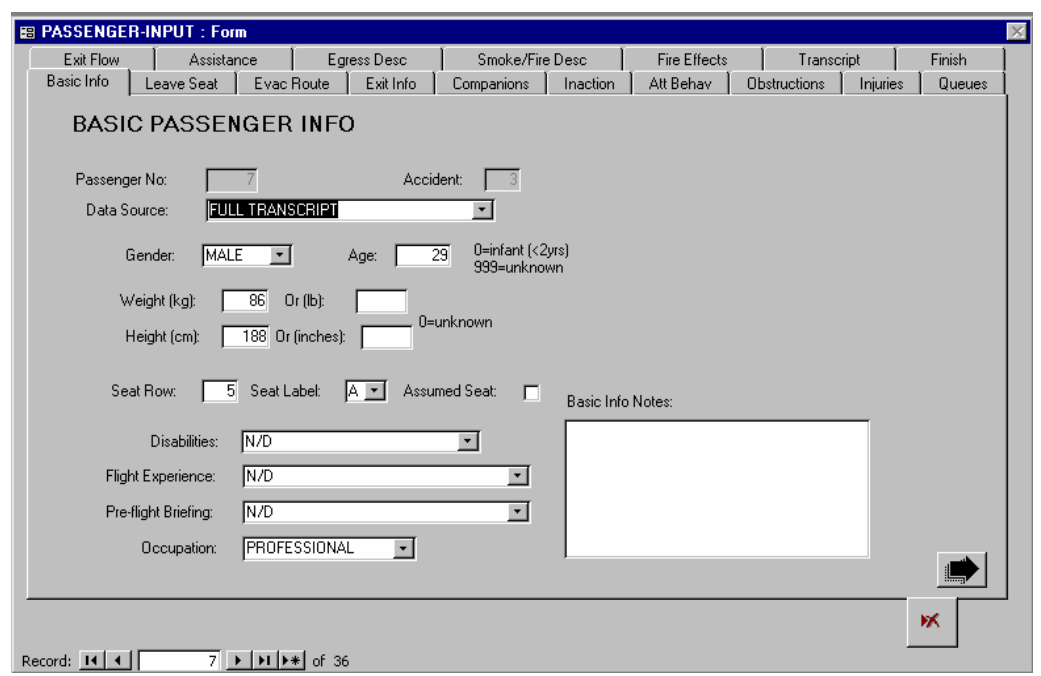

\section{Figure 1: The collection of forms making up the Passenger Component, highlighting the "Basic Info form".}

\subsection{Seat Plan Viewer}

Once the accident details are put into the database the layout of the cabin is usually available. The Seat Plan Viewer provides a quick diagrammatic version of the cabin enabling better visualisation of passenger starting location and exit use during the evacuation. 


\subsection{AASK V3.0 Query Engine.}

AASK V3.0 can be used in three modes, standalone on a single computer, over a local area intranet and over the internet. The same user interface is used for all three modes of operation. The user interface developed for AASK has been designed so that users without a detailed understanding of the ACCESS database - on which AASK is based can easily make use of the data. It should be stressed however that to run meaningful queries the user must understand the nature of the data held in the database.

The AASK database query applet enables authorised users to construct queries and to copy and paste query results into other analysis tools such as speadsheets. The user interface allows operations such as selection of query fields, inclusion of query condition/s and query field sorting. The AASK database query applet translates user queries based on the selected database fields, conditions, sorting etc into Structured Query Language (SQL). The SQL query is then sent to the AASK database and the results are displayed to the user.

Security of the database is maintained at a number of different levels with passwords for the software and control of machine access. Currently only users authorised by the CAA are given internet access to AASK V3.0. Those interested in using AASK may register at the site http://fseg.gre.ac.uk/aask/index.html.

\section{ANALYSIS OF DATA FROM AASK V3.0}

The AASK database can be used for a variety of purposes. The type of analysis performed is dependent on the nature of the questions posed to the database. Thus, the uses of AASK are far greater than those originally envisaged by its developers. In this section several analyses performed using the AASK database will be presented. These have been chosen to represent different levels of complexity in order to illustrate the depth and variety of the analysis possible using the AASK system. However, all analysis and results must be carefully considered within the context of the database. As an example the reactions of passengers to cabin crew commands might have an element of bias when it is recalled that all the passenger accounts are from those passengers who "responded" to the request for information. Finally, the initial analysis conducted in this section is identical to that undertaken using the previous version of the database [1,2]. This is undertaken to ensure that the conclusions previously drawn are still valid with the enlarged data set.

\subsection{Survivor and reply rate Analysis}

Of the 55 accidents, 31 were found suitable for this analysis as not all accidents in the database have individual survival accounts. The reply rate varies from $2.63 \%$ to $95.15 \%$. The average reply rate is $49.81 \%$, which means that we have data on approximately half the survivors of these accidents. The differences between the reply rates and the passengers entered can be accounted for in the inferred data particularly for children and travelling companions. 
This analysis, while quite simple, illustrates some of the benefits and dangers of using this type of system. This simple query allows an overview of the data available for analysis. The response to the enquiry is precise and was obtained in seconds. A traditional approach to retrieving this type of information from a large source of data would have taken quite some time and subject to errors. However, the results of the analysis must be tempered with the knowledge of the question posed. This data is biased as only those accidents where individual accounts are available are included, suggesting that a fairly high survival rate would be required in order to generate enough replies.

\subsection{Age and Gender Distribution}

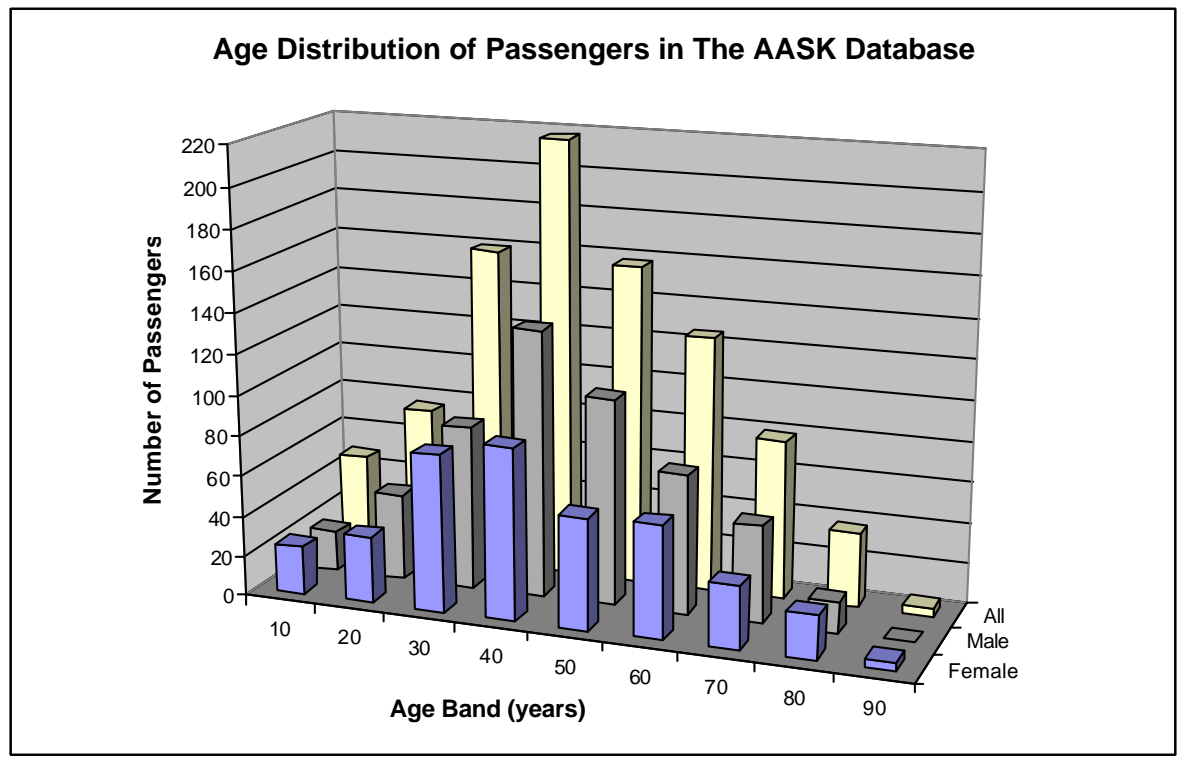

Figure 2: Age distribution of passengers in the AASK database

In addition to the previous type of analysis, individual passenger attributes may also be examined. For example, consider the age of the survivors. While this information is not known for all the survivors, the distribution of known ages is depicted in Figure 2. The information can be further categorised by other passenger attributes, such as gender. Analysis of the data reveals that the average age of all the 905 survivors in the database (where age is known) is 39.4 years, and that the average age of the males is 39.8 years while that of the females is 39.3 years.

\subsection{Nearest Exits Usage}

Within the aviation community it was a commonly held belief that most of passengers evacuate via their most familiar exit, thereby ignoring closer but unfamiliar emergency exits. Analysis using the earlier version of the AASK database [1,2] suggested that this was not the case and that overwhelmingly, passengers tended to use their nearest serviceable exit. The results from the analysis using the expanded AASK database confirm this observation with more than $70 \%$ of passengers who report their exit usage 
making use of the nearest available exit, 619/958. (the figure after the "/" include passengers where exit use and/or seat location has been inferred).

Of the 179/304 passengers that did not use their nearest exit, 103/142 passengers supplied reasons for their actions. and these are shown in Table 1 . While by no means complete, this analysis suggests that an overwhelming $88 \%$ of those passengers reporting their exit usage, either used or had a good reason not to use their nearest exit. (If the passengers with inferred data is included this figure becomes $83 \%$ ). The remaining $12 \% / 17 \%$ did not supply any reason for not using their nearest exit, however, this is not to say that they did not have a reason

Table 1: Reasons for exit choice given by those passengers NOT using nearest exit. Note values after the " $/$ " include pax where exit use and/or seat location is inferred.

\begin{tabular}{|l|c|}
\hline Reason For Exit Choice & Number of Pax \\
\hline No Data & $75 / 160$ \\
\hline Not Applicable (e.g. rescued) & $1 / 2$ \\
\hline NEAREST EXIT WAS/BECAME UNAVAILABLE & $27 / 39$ \\
\hline FOLLOWED ATTENDANT INSTRUCTIONS & $22 / 29$ \\
\hline FOLLOWED OTHER PAX & $17 / 24$ \\
\hline SHORTER QUEUE THAN OTHER EXITS & $11 / 14$ \\
\hline CHOICE MADE BEFORE EGRESS & $9 / 11$ \\
\hline NEAREST EXIT & $7 / 7$ \\
\hline FOUND EXIT DURING EGRESS & $7 / 10$ \\
\hline FOLLOWED EMERGENCY LIGHTS & $1 / 1$ \\
\hline ONLY AVAILABLE EXIT & $1 / 1$ \\
\hline FOLLOWED COMPANION & $1 / 1$ \\
\hline HELPED THROUGH EXIT & $0 / 5$ \\
\hline
\end{tabular}

\subsection{Direction and distance travelled}

It is also interesting to consider the direction travelled by the passengers when evacuating. It was found that of the $619 / 958$ passengers for which we know the direction of travel, 63\% / 62\% travelled forward, 31\% / 34\% travelled towards the rear while the remainder were situated within an exit row (again the figures after the "/" include passengers where exit use and/or seat location has been inferred). This may suggest that the passengers have a propensity for travelling forward.

However, of those passengers choosing to travel forward, 70\% / 65\% have selected their nearest exit, while for those choosing to travel towards the aft, $69 \% / 71 \%$ have selected their nearest exit. This suggests that the overriding ambition of the passengers is to exit via their nearest exit, rather than to travel forward. In addition, this suggests that exit selection is based on a rational decision, at least for the survivors.

The mean distance travelled (in terms of seat rows) by survivors in evacuating is $6.3 / 7.3$ seat rows. Furthermore, those passengers who select their nearest exit - excluding those in exit rows - travel approximately 3.8/4.5 seat rows regardless if they travel forward or aft, while those who do not travel towards their nearest exit travel at least three times as far. 


\subsection{Exit distribution}

As an extension to the previous analysis, it is possible to examine the exit usage in terms of exit location. This analysis is restricted to aircraft with three exit pairs where at least one exit from each pair was available. This was compared with the results from two equivalent aircraft evacuation certification trials.

This analysis, shown in Table 2 suggests that a bias in exit usage exists for those in the middle section of the cabin. The observed bias remains even if the third accident - which only has a passenger loading of $39 \%$ - is removed from the sample. This is a disturbing trend as these exits are the smaller TYPE-III passenger operated hatch exits.

Table 2: Exit usage in terms of percentage of passengers using each generalised exit position. Information in brackets identifies exit type.

\begin{tabular}{||c|c|c|c|c||}
\hline \hline Accident Aircraft & Pax Loading & Fwd (\%) & Mid (\%) & Aft (\%) \\
\hline 1 & $93.6 \%$ & $19.2[\mathrm{I}]$ & $61.5[\mathrm{III}]$ & $19.2[\mathrm{I}]$ \\
\hline 2 & 96.6 & $39.5[\mathrm{I}]$ & $37.2[\mathrm{III}]$ & $23.3[\mathrm{I}]$ \\
\hline 3 & $39.0 \%$ & $44.7[\mathrm{I}]$ & $50.0[\mathrm{III}]$ & $5.3[\mathrm{I}]$ \\
\hline Mean (\%) & - & $\mathbf{3 4 . 5}$ & $\mathbf{4 9 . 6}$ & $\mathbf{1 5 . 9}$ \\
\hline \hline Certification Aircraft & & & & \\
\hline 1 & & 40 & 20 & 40 \\
\hline 2 & & 27 & 37 & 36 \\
\hline Mean (\%) & & $\mathbf{3 3 . 5}$ & $\mathbf{2 8 . 5}$ & $\mathbf{3 8}$ \\
\hline
\end{tabular}

Exit usage for two aircraft with three exit pairs, derived from actual 90 -second certification trials, suggests that the exit usage achieved in certification trials is quite different from that in actual accidents. In actual accidents, there appears to be a biased trend for exit usage in the midsections (i.e. the nearest exit for the majority of passengers) of the aircraft. Yet in the certification trials, the mean load on each exit pair is far more even and furthermore, fewer passengers use the midsection exits, the reverse of that seen in actual accidents.

The most probable reason for this lies in the behaviour of the passengers. Essentially, in a real accident the passengers have a higher motivation to escape and tend to do so by what they perceive to be the most direct method - their nearest exit. The cabin crew procedures used in certification trials work quite well and achieve a well balanced evacuation with most of the exits working in an efficient manner. However, in these circumstances, the passengers are working in a highly co-operative manner as opposed to the competitive behaviour likely to be exhibited by passengers in life threatening situations. This suggests that formulating cabin crew procedures on the basis of certification experience may be misleading in terms of their actual effectiveness.

\subsection{Exit availability}

In this analysis, the exits that are actually available during the accident are examined. The accidents used in this summary ignore all those where the aircraft landed in water or substantial damage occurred to the aircraft fuselage, i.e. where significant breaks in the fuselage occurred, and include only those accidents where information is known about all the exits. As a result, seven accidents were selected. It should be noted here that the 
criteria used for including aircraft in the analysis has been tightened since the previous analysis [1,2] and all the cases included here have a strict arrangement of exit pairs in forward, mid and aft positions (i.e. three exit pairs).

The frequency of exit availability for the aircraft involved in these seven accidents is displayed in Table 3. At the FWD generalised location, one exit is available in only $14.3 \%$ of cases while both exits are available $71.4 \%$ of the time. In the case of MID positioned exits, the results are identical, in most cases ( $71.4 \%$ of the time) both exits are available. Finally, the AFT positioned exits show that having both or a single exit available are equally likely (42.9\%).

As part of the 90-second certification exercise, the trial criteria stipulate that only half of the available exits can be used. Without exception, where aircraft have exit pairs, only one exit of each pair is selected. If this scenario represented reality we would expect to see the highest percentages in the "One Exit" column of Table 3. Thus, the exit configuration used in the 90 second certification exercise does not correspond to the exit availability suggested in the sample of real accidents contained in AASK V3.0. Furthermore, the exit configuration actually used in the 90 -second certification exercise is not a particularly onerous configuration as an exit is available in each cabin section. A more challenging exit combination - while maintaining the $50 \%$ condition - that is also consistent with the observed exit availability would involve both FWD exits and a single AFT exit, or both MID exits and a single AFT exit.

Once again it is important to note that results presented in Table 3 only refer to seven accidents and so is by no means complete. Furthermore, the analysis only considers the frequency of availability of exits within an exit pair. It does not consider which exit combinations across exit pairs are likely.

Table 3: Proportion of exit availability in terms of generalised exit positions for three-exit pair aircraft.

\begin{tabular}{|c|c|c|c|}
\hline & \multicolumn{3}{|c|}{ Availability (\%) of exit in exit pair. } \\
\hline Exit Position & No Exits & \multicolumn{1}{|c|}{ One Exit } & Both Exits \\
\hline FWD & $14.3 \%$ & $14.3 \%$ & $71.4 \%$ \\
\hline MID & $14.3 \%$ & $14.3 \%$ & $71.4 \%$ \\
\hline AFT & $14.3 \%$ & $42.9 \%$ & $42.9 \%$ \\
\hline
\end{tabular}

\section{ADDITIONAL ANALYSIS}

The analysis presented above was an extension of previous studies undertaken with the expanded AASK database. In this section we consider several new analyses. The results presented here are in summary form, a fuller account of this work may be found in [9].

\subsection{Seat belt difficulty}

In aircraft evacuations the response time of passengers tends to be relatively short, as there is a high degree of apparent awareness of the seriousness of the incident. However, some passengers are unable to commence their evacuation due to difficulties leaving their seat, either due to the aisle being full of passengers, or simply because they had difficulties releasing their seatbelt. In this example analysis, the latter of these cases is 
investigated. The database was queried to find those passengers who had mentioned difficulties with seat belts. In the earlier study only 44 passengers had reported difficulties with their seat belts, whereas in the present analysis some 81 passengers have reported difficulties.

The passenger seat belt difficulties analysis is broken down into three categories, those passengers who helped others, those passengers who received assistance and those passengers who managed alone. The number of passengers in these categories is such that statistical analysis in possible. From the initial analysis it appears that there is a difference in the observed genders of passengers in these categories and those expected from the distribution of the general database population. Figure 3 shows the categories and results graphically.

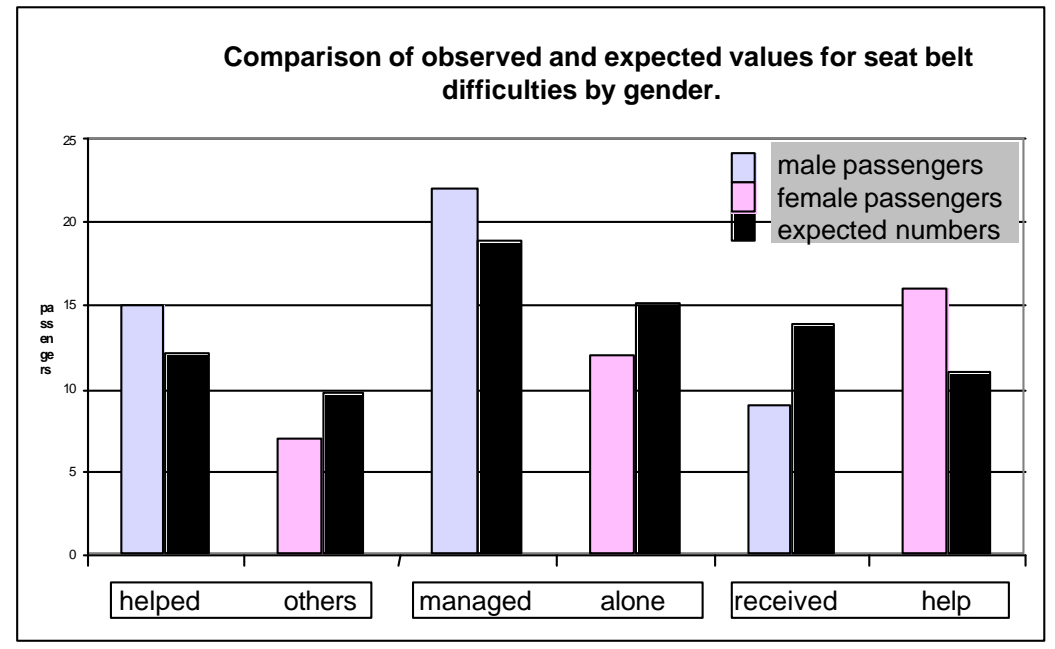

Figure 3: Breakdown of number of passengers experiencing s eatbelt difficulty.

From this distribution it is clear that males have fewer problems with seat belts than females and that males are also more likely to render assistance to others than females. Furthermore, the number of males who rendered assistance or who managed alone is more than would be expected from the overall gender proportions. Similarly, the number of females who managed alone or who helped others is significantly less than would be expected. Finally, the number of males who received help is significantly less than would be expected from the overall gender mix while the number of females who received help is significantly greater.

It is possible to further refine the analysis of the 81 seat belt difficulty cases. It is important to consider whether the occurrence of difficulties experienced by passengers in actually releasing the seat belt is related to the age of the individual concerned. This analysis requires that the passengers in the first category are removed from consideration. The remaining records must be further sorted with reference to the field "seat belt info". From this analysis, three categories of seat belt difficulty were accepted for consideration. These are:

\section{Unfamiliar with buckle release mechanism:}




\section{e.g. "It took him 5 to 6 seconds to determine how to undo his seat belt."}

\section{Environmental related complications excluding immersion in water:}

e.g. "could not release seat belt due to smoke reduced visibility problems. Erroneously tugged on the buckle instead of undoing it"

\section{Buckle location:}

e.g. "thought seat belt buckle was at side as in a car not in centre."

Using these criteria, the number of passengers experiencing difficulty with seat belt release is reduced to 27 . The age distribution of passengers experiencing difficulties with releasing the seat belt was investigated. While the general database population distribution is positively skewed towards younger passengers the distribution for those involved with seatbelt difficulties is negatively skewed towards older passengers. The distribution reveals that older passengers appear to be more likely to experience difficulties with seat belts than younger passengers.

\subsection{Exit availability}

Extending the work described in section 4.5 and 4.6, overall exit availability for aircraft with three and four exit pairs potentially available were considered. Accidents were restricted to situations in which the fuselage did not rupture and the aircraft was not partially or totally immersed in water. From the database five aircraft were found to be suitable with three exit pairs and 12 with four exit pairs.

For the aircraft with three exit pairs, one aircraft had exactly $50 \%$ of the exits available and one aircraft had less than $50 \%$ of the exits available. For the aircraft with four exit pairs, two aircraft had exactly $50 \%$ of the exits available and two aircraft had less than $50 \%$ of the exits available. Thus out of the 17 accidents considered, six (i.e. $35 \%$ ) had half or less of the potentially available exits usable. Furthermore, of the 17 aircraft considered, 10 or $59 \%$ had a cabin section in which no exits were available. No cases were found in which a single exit from an exit pair was available throughout the cabin. This result is identical to an earlier study by Fons Schaeffer in which he examined accidents from 1960 to 1989 [11] (the current study investigates accidents from 1982 1998). As noted earlier, the 90 second certification trial makes use of a situation in which $50 \%$ of the exits are available and that one exit from each exit pair is available.

\subsection{Nearest Exit Usage: a comparison between accident and trial data.}

In section 4.3 we noted that $88 \%$ of the passengers in accidents made use of their nearest exit or had a good reason for not using their nearest exit. To compare this with the situation in certification trials, data from 18 past certification trials (12 wide body and 6 narrow body) [10] was entered into AASK and the nearest exit usage analysis was repeated. The analysis revealed that on average, $76 \%$ of the passengers in certification trials make use of their nearest exit. Only in two of the 18 trials did we find that the nearest exit usage was greater. In certification trials, cabin crew will often direct passengers away from their nearest exit. This would lead to the reduced number of passengers making use of their nearest exit in certification trials. In contrast, in actual aircraft evacuation emergencies, the $88 \%$ figure is made up of passengers who used their nearest exit or had a very good reason not to use their nearest exit e.g. exit blocked. However, in this figure we have included those passengers who were redirected by cabin 
crew. In order to be consistent with the certification trial figure, these people should be removed from the total. If this is done the figure is reduced to $84 \%$. Even with these adjusted figures, passengers tend to use their nearest serviceable exits more often in real accident scenarios than we find in certification tests.

\section{CONCLUSIONS}

The AASK database provides a versatile aid in the analysis of human behaviour during aircraft evacuation. While much data exists for input to the database, the data is limited in scope in that the qualitative aspects of the data far outweigh the quantitative. As such, conclusions drawn from the database must be treated with caution and with full knowledge of the implications of the questions posed and the nature of the data used to provide the responses.

With the development of AASK V3.0, it is now possible to access detailed survivor (passenger and crew) information as well as data for all fatalities documented in the accident reports. The cabin crew component has become a significant aspect of the database providing insight into cabin conditions and passenger behaviour as seen from professionally trained cabin specialists. The Seat Plan Viewer graphically displays the starting locations of all the passengers - both survivors and fatalities - as well as the exits used by the survivors.

The database is proving very useful as a development tool for evacuation models such as airEXODUS. In addition, AASK is shedding light on what really happens during aircraft emergency evacuations and as such is helping to dispel some of the myths that pervade aviation safety. Detailed analysis of past accident scenarios and the human behaviour that results could be used to help define more representative certification scenarios to be used in a risk analysis approach to performance based safety analysis. This type of analysis is vital if trends in passenger behaviour are to be understood and ultimately used to improve passenger safety.

Through support from the UK CAA, work on AASK is continuing. This includes the inclusion of additional accident data, the further development of the user interface and finally additional data analysis. Answers to questions concerning exit usage, behaviour in poor visibility, the role of the Cabin Crew and the seating of infants are just some of the issues that are to be addressed using the database. AASK V3.0 can be accessed over the internet. Those interested in using AASK may register at the site http://fseg.gre.ac.uk/aask/index.html. The developers are hoping to enlist the assistance of cabin safety specialists in the further development and improvement of the AASK database.

\section{ACKNOWLEDGEMENTS}

The authors are indebted to the UK CAA for their financial support of this project, and also to the NTSB and the AAIB for their support in providing the data. Finally, Professor E R Galea is indebted to the UK CAA for their financial support of his personal chair in Mathematical Modelling at the University of Greenwich. 


\section{REFERENCES}

1. Owen, M., Galea, E.,R., Lawrence, P.,J., and Filippidis, L. AASK - Aircraft Accident Statistical Knowledge: A Database of Human Experience in Evacuation Derived from Aviation Accident Reports, Human Behaviour in Fire, Proc, $1^{\text {st }}$ Int Symp, Ed: J.Shields, NI, pp 509- 518, 1998.

2. Owen, M., Galea, E.,R., Lawrence, P.,J., and Filippidis, L. AASK - Aircraft Accident Statistical Knowledge: A Database of Human Experience in Evacuation Reports, The Aeronautical Journal of the Royal Aeronautical Society, August/September 1998, pp 353- 363.

3. Galea, E.R., Cooney, D., Dixon, A.J.P., Finney, K., and Siddiqui, A. THE AASK DATABASE: Aircraft Accident Statistics and Knowledge. A Database to Record Human Experience of Evacuation in Aviation Accidents. Report for CAA Project 277/SRG/R+AD, April 2000.

4. Galea, E. R., and Galparsoro, J. M. P., 1994, A Computer Based Simulation Model for the Prediction of Evacuation from Mass Transport Vehicles Fire Safety Journal, 22 pp 341 366.

5. Galea, E. R., Owen, M., and Lawrence, P., 1996, Computer Modelling of Human Behaviour in Aircraft Fire Accidents, Toxicology, Vol. 115, Nos. 1-3, 63-78.

6. Owen, M., Galea, E.R., Lawrence, P.J. and Filippidis, L. The Numerical Simulation Of Aircraft Evacuation And Its Application To Aircraft Design And Certification. The Aeronautical Journal Of The Royal Aeronautical Society, June/July 1998, pp 301-312.

7. Blake, S., J., Galea, E., R., Gwynne, S., Lawrence, P.J. and Filippidis, L. "Examining The Effect of Exit Separation On Aircraft Evacuation Performance Using Evacuation Modelling Techniques: 'Is The 60 Foot Rule Relevant?'” University of Greenwich CMS Press Paper No. 01/IM/71, ISBN 1899991727, 2001

8. Civil Aviation Authority, Survivable Accidents Database, Software V2.0, Data Issue 8, 1998.

9. Galea, E.R., Finney, K.M., Dixon, A.J.P., Cooney, D. P., and Siddiqui, A., “An analysis of human behaviour during aircraft evacuation situations using the AASK V3.0 database". Paper in preparation, 2002.

10. Owen, M., Galea, E.R., Dixon A.J.P., 90-second Certification Trial Data Archive Report, Confidential, Prepared for U.K. CAA for project 049/SRG/R\&AD, March 1999.

11. Schaeffer, F., Passenger Emergency Exit Usage in Actual emergencies of Jet Airliners 1960 - 1989., Proceedings European Cabin Safety Conference 1990, CAP 583, pp 68-89, 1990. 